\title{
Perfil de pacientes com câncer de laringe atendido nos hospitais de alta complexidade oncológica no Pará na série temporal 2000-2017
}

Profile of laryngeal cancer patients attended in hospitals of high oncological complexity of Pará in the time series 2000-2017

Perfil de los pacientes con cáncer de laringe atendidos en hospitales de alta complejidad oncológica de Pará en la serie temporal 2000-2017

Jaqueline Dantas Neres Martins ORCID: https://orcid.org/0000-0002-3377-9482

Universidade do Estado do Pará, Brasil. E-mail: jaqueline170896@gmail.com

Dayara Nazaré Rosa de Carvalho ORCID: https://orcid.org/0000-0001-8569-3392

Universidade do Estado do Pará, Brasil

E-mail: dayara_twain@ @otmail.com

Dandara de Fátima Ribeiro Bendelaque ORCID: https://orcid.org/0000-0002-5580-284X Universidade Federal do Pará, Brasil E-mail: bendelaqued@gmail.com

Marcela Raíssa Asevedo Dergan ORCID: https://orcid.org/0000-0003-1457-0242 Universidade do Estado do Pará, Brasil E-mail: derganm20@gmail.com

Susi dos Santos Barreto de Souza ORCID: https://orcid.org/0000-0002-4138-7147

Universidade do Estado do Pará, Brasil

E-mail: susis.barreto@yahoo.com.br Lucas de Jesus Pereira ORCID: https://orcid.org/0000-0002-3938-3286 Hospital Universitário Lauro Wanderley, Brasil E-mail: lucas-laje@hotmail.com

Renata Ferreira Magno ORCID: https://orcid.org/0000-0003-3048-2760 Universidade da Amazônia, Brasil E-mail: renattamagno@gmail.com

Lucrecia Aline Cabral Formigosa ORCID: https://orcid.org/0000-0003-4245-672X Universidade Federal do Pará, Brasil E-mail: lucrecia_cabral@hotmail.com

Lourrany Kathlen Barbosa Fernandes Dias ORCID: https://orcid.org/0000-0001-6460-7774 Universidade da Amazônia, Brasil

E-mail: lourrany.barbosa08@gmail.com

Larissa Graziela Sousa da Silva ORCID: https://orcid.org/0000-0002-5853-334X Hospital Universitário Getúlio Vargas, Brasil E-mail: laragazi@gmail.com

Mainessa da Guia Rodrigues ORCID: https://orcid.org/0000-0003-3867-8995

Universidade Federal do Amazonas, Brasil

E-mail: mainessarodrigues@hotmail.com

Karina Teixeira Dutra ORCID: https://orcid.org/0000-0002-4828-8256 Faculdade Paraense de Ensino, Brasil E-mail: karinadutra26@gmail.com

Tainan Fabrício da Silva ORCID: https://orcid.org/0000-0001-6761-1365

Universidade Federal do Amazonas, Brasil

E-mail: tainanfabricio@hotmail.com 


\author{
Natalie Kesle Costa Tavares \\ ORCID: https://orcid.org/0000-0002-0482-6999 \\ Universidade Federal do Amazonas, Brasil \\ E-mail: natalietavares.nt@gmail.com \\ Paula Andreza Viana Lima \\ ORCID: https://orcid.org/0000-0002-8217-8288 \\ Universidade Federal do Amazonas, Brasil \\ E-mail: paulaviana_lima@hotmail.com \\ Rodrigo Damasceno Costa \\ ORCID: https://orcid.org/0000-0002-1442-001X \\ Distrito Sanitário Especial Indígena Médio Rio Purus, Brasil \\ E-mail: rodrigo-damasceno@outlook.com \\ Ingrid Melo de Menezes \\ ORCID: https://orcid.org/0000-0001-9249-9141 \\ Faculdade Integrada Brasil Amazônia, Brasil \\ E-mail: ingridmelooenfr@gmail.com \\ Djenanne Simonsen Augusto de Carvalho Caetano \\ ORCID: https://orcid.org/0000-0001-7987-3178 \\ Universidade do Estado do Pará, Brasil \\ E-mail: djenanne.caetano@uepa.br \\ Francinéa de Nazaré Ferreira de Castilho \\ ORCID: https://orcid.org/0000-0002-9278-0010 \\ Universidade do Estado do Pará, Brasil \\ E-mail: francineacastilho@hotmail.com \\ Viviane Ferraz Ferreira de Aguiar \\ ORCID: https://orcid.org/0000-0003-3025-1065 \\ Universidade Federal do Pará, Brasil \\ E-mail: viviane.ferraz29@gmail.com
}

\begin{abstract}
Resumo
Objetivo: Traçar o perfil de pacientes com neoplasia primária de laringe em hospitais de alta complexidade oncológica no Pará na série temporal 2000-2017. Método: Estudo epidemiológico, retrospectivo realizado com a base de dados secundários provenientes do Registro Hospitalar de Câncer (RHC). Resultados: o perfil de pacientes com neoplasia maligna da laringe em serviços de alta complexidade oncológica no estado do Pará são em sua maioria: sexo masculino, maior de 40 anos, histórico de etilismo e/ou tabagismo, branco ou pardo, ensino fundamental incompleto, diagnosticados nos estágios mais avançados. Conclusão: Dessa forma, percebe-se que esta neoplasia está fortemente alicerçada na interação dos fatores ambientais e endógenos. Sobretudo, o sistema de saúde, ainda é tênue para atender aos pacientes em risco ou com a neoplasia de laringe, a começar pelos índices altos de desconhecimento presente nas bases de dados secundários sobre indicadores em saúde, o que implica na tendência de certas variáveis importantes para a predição do câncer.
\end{abstract}

Palavras-chave: Neoplasias Laríngeas; Serviço hospitalar de oncologia; Sistema de saúde.

\begin{abstract}
Objective: To trace the profile of patients with primary laryngeal neoplasia in hospitals of high oncological complexity in Pará in the time series 2000-2017. Method: Retrospective epidemiological study conducted with the secondary database from the Hospital Cancer Registry (RHC). Results: the profile of patients with malignant laryngeal neoplasia in services of high oncological complexity in the state of Pará are mostly: male, over 40, history of alcoholism and/or smoking, white or brown, incomplete elementary school, diagnosed in the most advanced stages advanced. Conclusion: Thus, it is clear that this neoplasm is strongly based on the interaction of environmental and endogenous factors. Above all, the health system is still tenuous to serve patients at risk or with laryngeal neoplasia, starting with the high levels of ignorance present in the secondary databases on health indicators, which implies the tendency of certain important variables for cancer prediction.
\end{abstract}

Keywords: Laryngeal neoplasms; Oncology service hospital; Health systems.

\title{
Resumen
}

Objetivo: rastrear el perfil de pacientes con neoplasia laríngea primaria en hospitales de alta complejidad oncológica en Pará en la serie temporal 2000-2017. Método: estudio epidemiológico retrospectivo realizado con la base de datos secundaria del Hospital Cancer Registry (RHC). Resultados: el perfil de los pacientes con neoplasia laríngea maligna en servicios de alta complejidad oncológica en el estado de Pará son principalmente: hombres, mayores de 40 años, antecedentes de alcoholismo y / o tabaquismo, escuela primaria incompleta blanca o marrón, diagnosticados en las etapas más avanzadas avanzado. Conclusión: Por lo tanto, está claro que esta neoplasia se basa fuertemente en la interacción de factores ambientales y endógenos. Sobre todo, el sistema de salud sigue siendo frágil para atender a pacientes en riesgo o con neoplasia laríngea, comenzando con los altos niveles de ignorancia presentes en las bases de datos secundarias sobre indicadores de salud, lo que implica la tendencia de ciertas variables importantes. para la predicción del câncer.

Palabras clave: Neoplasias Laríngeas; Servicio de oncología en hospital; Sistemas de salud. 


\section{Introdução}

O câncer de laringe é um dos principais tipos de tumores no Brasil, consoante a isso, as estatísticas governamentais de incidência para o País no triênio 2020-2022 estimado por sexo, em homens, será de 6.470 casos e 1.180 para mulheres. O ranking desse tumor nos próximos anos para cada região será: Região centro-oeste e nordeste ( $8^{\circ}$ posição); Sudeste e norte $\left(9^{\circ}\right.$ posição); e Sul (10 posição) (Instituto Nacional de Câncer, 2019).

Equiparado a incidência, a mortalidade por esse tipo de câncer tem sido crescente nos últimos anos, com $4.382 \mathrm{em}$ 2016 e 4.501 em 2017, totalizando 73.946 óbitos nos anos 1996-2017 (DATASUS). Concomitante as estimativas relacionadas à patologia e seu prognóstico, os fatores causais da doença são relevantes para formular estratégias de prevenção, controle e manejo clínico no diagnóstico e tratamento do paciente (Deng et al.,2017).

Um dos principais causadores da neoplasia de laringe é a interação de fatores ambientais, individuais e coletivos, tais como o tabagismo, etilismo, infecção viral, sedentarismo, dislipidemia, hiperlipidemia, alimentação inadequada e determinadas exposições ocupacionais. A maioria da população tem pelo menos um desses fatores de risco e, portanto, está vulnerável ao mesmo (Deng et al.,2017).

As sintomatologias do agravo geralmente são rouquidão, odinofagia, disfagia e, em alguns casos, dificuldade respiratória. É importante salientar a relevância das sintomatologias, na qual suas apresentações por serem inespecíficas mimetizam e/ou podem dar outra impressão diagnóstica, contribuindo assim no retardo do diagnóstico e progressão do agravo e maiores chances de complicações e terapias mais fortes e imunossupressoras (Shephard, Parkinson \& Hamilton, 2019).

Há que ressaltar também que o sistema de saúde por vezes é seletivo e não consegue abarcar todos os pacientes com as sintomatologias, com necessidade de consulta com especialistas e/ou necessidade de tratamento limitando o acesso aos serviços de saúde (Deng et al.,2017; Shephard, Parkinson \& Hamilton, 2019). Dessa forma, diante desse arcabouço ressalta-se a importância de estudos epidemiológicos de perfis para traçar e possibilitar conhecimento científico importante para repensar as práticas assistenciais que facilitem o acesso e priorizem populações específicas e mais vulneráveis ao adoecimento por câncer de laringe.

Portanto, o presente trabalho tem como objetivo traçar o perfil de pacientes com neoplasia primária de laringe em hospitais de alta complexidade oncológica no Pará, na série temporal 2000-2017.

\section{Materiais e Método}

Trata-se de estudo quantitativo, epidemiológico, retrospectivo realizado no mês de abril de 2020, com a base de dados secundários provenientes do Registro Hospitalar de Câncer (RHC). Esse sistema permite extrair dados de pacientes com diagnóstico de câncer proveniente de hospitais de alta complexidade em oncologia de todos os estados. Sendo indispensável para avaliação da qualidade dos serviços prestados nestas instituições como subsídio para a tomada de decisões, gestão de qualidade e melhora da assistência em oncologia (Brasil, 2019; INCA, 2010).

Para a pesquisa foram selecionados casos de neoplasias malignas primárias da laringe, na série temporal 2000-2017, atendidas no Pará. Para este estudo foram utilizados as seguintes variáveis: sociodemográficas (sexo, faixa etária, raça/cor, escolaridade e estado conjugal); Hábitos/fatores de risco (ocupação, tabagismo, etilismo, histórico de câncer); relacionadas com internação e tratamento prévio ou atual (base do diagnóstico, estadiamento, diagnóstico e tratamento anterior; primeiro tratamento; resultado do $1^{\circ}$ tratamento, razão para não tratar). 


\section{Resultados}

Foram identificados 910 casos de neoplasia maligna de laringe no Pará nos anos 2000-2017, com destaque para o maior número de casos no sexo masculino representando 85,4\%. Em relação a faixa etária, predominou a de 60 a 69 anos (31,7\%), indivíduos de raça parda (34,7\%), pouca escolaridade, e estado conjugal casado. Conforme ilustrado na Tabela 1.

Tabela 1: Números de câncer de laringe por sexo, faixa etária, raça/cor, escolaridade e estado conjugal, ano 2000-2017.

\begin{tabular}{|c|c|c|}
\hline \multirow{2}{*}{ Variáveis } & \multicolumn{2}{|c|}{ Total $n=910$} \\
\hline & $\mathrm{n}^{\mathbf{0}}$ & $\%$ \\
\hline \multicolumn{3}{|l|}{ Sexo } \\
\hline Feminino & 132 & $14,6 \%$ \\
\hline Masculino & 778 & $85,4 \%$ \\
\hline \multicolumn{3}{|l|}{ Faixa etária } \\
\hline $00-09$ & 1 & $0,1 \%$ \\
\hline $10-19$ & 0 & $0 \%$ \\
\hline $20-29$ & 7 & $0,7 \%$ \\
\hline $30-39$ & 15 & $1,64 \%$ \\
\hline $40-49$ & 92 & $10,1 \%$ \\
\hline $50-59$ & 236 & $25,9 \%$ \\
\hline $60-69$ & 289 & $31,7 \%$ \\
\hline $70-79$ & 193 & $21,2 \%$ \\
\hline 80 ou mais & 156 & $17,1 \%$ \\
\hline Sem informação & 1 & $0,1 \%$ \\
\hline \multicolumn{3}{|l|}{ Raça } \\
\hline Raça Ignorada / em branco & 328 & $36 \%$ \\
\hline Branco & 231 & $25,3 \%$ \\
\hline Preta & 31 & $3,5 \%$ \\
\hline Amarelo & 4 & $0,4 \%$ \\
\hline Pardo & 315 & $34,7 \%$ \\
\hline Indígena & 1 & $0,1 \%$ \\
\hline \multicolumn{3}{|l|}{ Escolaridade } \\
\hline Ignorado / em branco & 98 & $10,8 \%$ \\
\hline Analfabeto & 151 & $16,6 \%$ \\
\hline Ensino Fund. Incompleto & 449 & $49,4 \%$ \\
\hline Ensino Fund. Completo & 112 & $12,3 \%$ \\
\hline Ensino Médio Completo & 83 & $9,1 \%$ \\
\hline Ensino Superior Incompleto & 2 & $0,2 \%$ \\
\hline Ensino Superior Completo & 15 & $1,6 \%$ \\
\hline \multicolumn{3}{|l|}{ Estado Conjugal } \\
\hline Ignorado & 33 & $3,7 \%$ \\
\hline Solteiro & 178 & $19,6 \%$ \\
\hline Casado & 498 & $54,7 \%$ \\
\hline Separado & 52 & $5,7 \%$ \\
\hline União estável & 53 & $5,8 \%$ \\
\hline Viúvo & 96 & $10,5 \%$ \\
\hline
\end{tabular}

Fonte: Integrador RHC (2020).

No que se refere ao número de casos ocupação, destacaram-se os trabalhadores agropecuários (12,5\%), condutores de veículo $(5,8 \%)$ e outras atividades agrícolas $(5,1 \%)$. Nota-se também que 45,9\% dos casos se referem a outras ocupações, conforme demonstrado na Tabela 2. 
Tabela 2: Número de casos por ocupação, ano 2000-2017.

\begin{tabular}{lcc}
\hline & \multicolumn{2}{c}{ Total n= 910 } \\
\cline { 2 - 3 } Variável & $\mathbf{n}^{\mathbf{0}}$ & $\mathbf{\%}$ \\
Trabalhadores agropecuários & 114 & $12,5 \%$ \\
Condutor de veículos & 53 & $5,8 \%$ \\
Outros trabalhadores agrícolas & 46 & $5,1 \%$ \\
Pedreiro/estucador & 27 & $3 \%$ \\
Pescador & 32 & $3,5 \%$ \\
Servidores edifícios & 15 & $1,7 \%$ \\
Carpinteiro & 14 & $1,6 \%$ \\
Doméstica & 14 & $1,6 \%$ \\
Comerciante & 18 & $1,9 \%$ \\
Ambulante/jornaleiro & 21 & $2,3 \%$ \\
Outras ocupações & 418 & $45,9 \%$ \\
Não se aplica & 73 & $8,1 \%$ \\
Sem informação & 65 & $2 \%$ \\
\hline
\end{tabular}

Fonte: Integrador RHC (2020).

Sobre a presença de fatores de risco associados ao câncer sobressaíram-se os números de presença de hábitos atuais ou histórico de tabagismo e etilismo, onde 332 eram etilistas (36,5\%) e 183 ex-consumidores (20,1\%). Sobre o tabagismo 489 indivíduos faziam uso do fumo $(53,8 \%)$ e 266 eram ex-consumidores $(29,3 \%)$. Conforme demonstra a Tabela 3.

Tabela 3: Distribuição de fatores de associados aos casos de câncer de laringe, ano 2000-2017.

\begin{tabular}{lcc}
\hline & \multicolumn{2}{c}{ Total n= 910 } \\
Variáveis & $\mathbf{n}^{\mathbf{0}}$ & $\mathbf{\%}$ \\
Etilismo & 3 & $0,3 \%$ \\
Não se aplica & 66 & $7,2 \%$ \\
Não avaliado & 332 & $36,5 \%$ \\
Sim & 158 & $17,4 \%$ \\
Não & 183 & $20,1 \%$ \\
Ex-consumidor & 168 & $18,5 \%$ \\
Sem informações & & \\
Tabagismo & 1 & $0,1 \%$ \\
Não se aplica & 10 & $1 \%$ \\
Não avaliado & 489 & $53,8 \%$ \\
Sim & 83 \\
Não & 266 \\
Ex-consumidor & 61 & $9,1 \%$ \\
Sem informações & & $29,3 \%$ \\
Histórico Familiar de Câncer & 131 \\
Sim & 200 & $6,7 \%$ \\
Não & 579 & $14,3 \%$ \\
Sem informação & $22 \%$
\end{tabular}

Fonte: Integrador RHC (2020).

Quanto à base do diagnóstico do tumor prevaleceu histologia do tumor primário com 881 casos (97\%). Sobre o estadiamento clínico do paciente, 106 apresentavam estágio 3 (11,7\%) e 102 estágio 4A (11,2\%). Sobre a chegada do paciente 
a instituição de saúde, 589 (64,8\%) chegaram com diagnóstico e sem tratamento, seguido por $113(12,4 \%)$ sem diagnóstico e sem tratamento, ambos demonstrados na Tabela 4.

Tabela 4: Estimativa da base diagnóstica, estadiamento e diagnóstico e tratamento anterior em pacientes com tumor primário de laringe, ano 2000-2017.

\begin{tabular}{lcc}
\hline & \multicolumn{2}{c}{ Total $\mathbf{n = 9 1 0}$} \\
\cline { 2 - 3 } Variáveis & $\mathbf{n}^{\mathbf{0}}$ & $\mathbf{\%}$ \\
Base do diagnóstico & 1 & $0,1 \%$ \\
Citologia & 10 & $1 \%$ \\
Clínica & 3 & $0,3 \%$ \\
Exame por imagem & 8 & $0,9 \%$ \\
Histologia da metástase & 881 & $97 \%$ \\
Histologia do tumor primário & 2 & $0,2 \%$ \\
Marcadores tumorais & 2 & $0,2 \%$ \\
Pesquisa clínica & 3 & $0,3 \%$ \\
Sem informação & & \\
Estadiamento TNM & 71 & $7,8 \%$ \\
1 & 71 & $7,8 \%$ \\
2 & 106 & $11,7 \%$ \\
3 & 1 & $0,1 \%$ \\
3B & 12 & $1,4 \%$ \\
4 & 102 & $11,2 \%$ \\
$4^{\text {a }}$ & 17 & $1,9 \%$ \\
4B & 10 & $1 \%$ \\
4C & 431 & $47,4 \%$ \\
Não se aplica & 89 & $9,7 \%$ \\
Sem informação & & \\
Diagnóstico e tratamento anterior & 113 & $12,4 \%$ \\
Sem diagnóstico e sem tratamento & 589 & $64,8 \%$ \\
Com diagnóstico e sem tratamento & 93 & $10,2 \%$ \\
Com diagnóstico e com tratamento & 2 & $0,2 \%$ \\
Outros & 113 & $12,4 \%$ \\
Sem informação & & \\
\hline & & \\
\hline
\end{tabular}

Fonte: Integrador RHC (2020).

Quanto aos fatores associados ao tratamento, a terapêutica mais utilizada foi à radioterapia 196 (21,6\%), cirurgia 134 (14,8\%), combinação de radioterapia com quimioterapia 132 (14,5\%) e nenhuma terapêutica 209 (23\%). Sobre o resultado do $1^{\circ}$ tratamento, 278 (30,5\%) apresentavam doença estável e 228 (25\%) doença em progressão. E as razões para não tratamento, $53(5,9 \%)$ casos foram por doença avançada e falta de condição, conforme ilustra a Tabela 5. 
Tabela 5: Fatores associados ao tratamento de câncer de laringe, ano 2000-2017.

\begin{tabular}{|c|c|c|}
\hline \multirow{2}{*}{ Variáveis } & \multicolumn{2}{|c|}{ Total $n=910$} \\
\hline & $\mathrm{n}^{\mathbf{0}}$ & $\%$ \\
\hline \multicolumn{3}{|l|}{$1^{\circ}$ Tratamento recebido } \\
\hline Cirurgia & 134 & $14,8 \%$ \\
\hline Quimioterapia (Qt) & 42 & $4,7 \%$ \\
\hline Radioterapia (Rxt) & 196 & $21,6 \%$ \\
\hline $\mathrm{Qt}+\mathrm{Rxt}$ & 132 & $14,5 \%$ \\
\hline Outros & 10 & $1 \%$ \\
\hline Cirurgia+ outros $+Q t+R x t$ & 1 & $0,1 \%$ \\
\hline Cirugia+ Qt & 20 & $2 \%$ \\
\hline Cirugia+ Qt+ Rxt & 70 & $7,7 \%$ \\
\hline Cirugia+ Rxt & 89 & $9,8 \%$ \\
\hline Outros+Qt & 2 & $0,2 \%$ \\
\hline Outros+ Qt+ Rxt & 1 & $0,1 \%$ \\
\hline Outros+ Rxt & 2 & $0,2 \%$ \\
\hline Nenhum & 209 & $23 \%$ \\
\hline Sem informações & 2 & $0,2 \%$ \\
\hline \multicolumn{3}{|l|}{ Resultado final do $1^{\circ}$ Tratamento recebido } \\
\hline Doença em progressão & 128 & $14 \%$ \\
\hline Doença estável & 278 & $30,5 \%$ \\
\hline Fora de possibilidade terapêutica & 21 & $2,4 \%$ \\
\hline Remissão parcial & 55 & $6 \%$ \\
\hline Sem evidência da doença (Remissão completa) & 53 & $5,9 \%$ \\
\hline Não se aplica & 228 & $25 \%$ \\
\hline Óbito & 80 & $8,8 \%$ \\
\hline Sem informações & 67 & $7,4 \%$ \\
\hline \multicolumn{3}{|l|}{ Razões para não tratar } \\
\hline Abandono do tratamento & 15 & $1,7 \%$ \\
\hline Complicações do tratamento & 1 & $0,1 \%$ \\
\hline Doença avançada, falta de condições clínicas ou outras & 53 & $5,9 \%$ \\
\hline Recusa do tratamento & 5 & $0,5 \%$ \\
\hline Tratamento realizado fora & 9 & $0,9 \%$ \\
\hline Óbito & 21 & $2,3 \%$ \\
\hline Não se aplica & 684 & $75,2 \%$ \\
\hline Sem informação & 115 & $12,7 \%$ \\
\hline Outras & 7 & $0,7 \%$ \\
\hline
\end{tabular}

Fonte: Integrador RHC (2020).

\section{Discussão}

Dessa forma, sobressaiu neste estudo o perfil de pacientes com neoplasia maligna da laringe: sexo masculino, maior de 40 anos, histórico de etilismo e/ou tabagismo, branco ou pardo, ensino fundamental incompleto, diagnosticado nos estágios mais avançados e que adentram no sistema de alta complexidade em oncologia com diagnóstico e sem tratamento na qual geralmente as terapias primárias são cirurgia ou radioterapia.

Estudos internacionais apresentam resultados semelhantes em relação a esse perfil, como por exemplo, em um estudo comparativo de análise clínica e imunohistoquímica realizado na Romênia, descreve que o perfil de paciente com esta neoplasia foi de $97 \%$ no sexo masculino, provenientes da área rural com 70\%, faixa etária entre 51-70 anos, 75\% diagnosticados em estágio IV e $23 \%$ em estágio III. Em outra pesquisa, realizada no Texas, identificou que a maioria era do 
sexo masculino74\%, média de idade 59 anos, 95\% fumante e 58\% tratados com quimioterapia (Ciolofan et al.,2017; Mulcahy, Mohamed, Kanwar, Hutcheson, Ghosh \& Vock, 2018).

Em uma pesquisa realizada com 48 registros de câncer de base populacional demonstrou que a incidência de câncer de laringe é 4 vezes maior em homens do que em mulheres (Perdomo, Roa, Brenan, Forman \& Sierra, 2016). Tanto este estudo como outros tem associado essa condição a presença dos principais fatores de risco para doença mais ocorrentes em homens, tais como o tabagismo e etilismo.

Em um estudo realizado com pacientes americanos e europeus, demonstrou que o tabagismo e o etilismo são um dos principais responsáveis pelos cânceres de cabeça e pescoço, principalmente os cânceres orofaríngeos. Ademais, observou-se que os homens os quais nutriam um destes hábitos isolados dispunham de maior risco de câncer de laringe e a associação dos dois despontavam o risco de adquirir a doença (Hashibe et al.,2009).

Alicerçando esse achado, outro estudo sobre os efeitos de cessar o hábito de fumar e consumir álcool, revelou que parar de fumar por 1-4 anos teve efeito benéfico, pois reduziu a probabilidade de ter cânceres de cabeça e pescoço atingindo até mesmo limiar e nível de significância semelhante ao de quem nunca havia fumado, em comparação, as pessoas que findavam com o etilismo reduziam as probabilidades de ocorrências cancerígenas, no entanto, os efeitos benéficos e proporção semelhante ao de quem nunca havia bebido ocorre em longo prazo (Perdomo, Roa, Brenan, Forman \& Sierra, 2016).

Em uma pesquisa realizada com pacientes asiáticos, demonstrou que o tabaco também foi fator de risco significativo para câncer de laringe, sendo a idade de início um fator importante no despontamento dos riscos, pois se observou que o risco era proporcionalmente maior em pessoas que iniciaram o fumo antes dos 18 anos Jayalekshmi, Nandakumar, Akiba, Gangadharan \& Koriyama, 2013).

A idade tem sido pertinente como vulnerabilidade para adquirir a doença demonstrado como fator significativo em pesquisas (Ciolofan et al.,2017; Mulcahy, Mohamed, Kanwar, Hutcheson, Ghosh \& Vock, 2018) aliado claramente a outros fatores já descrito neste estudo. A partir de 59 anos, nota-se o maior número de casos. A partir desta idade as médias de prevalência da doença tem sido variável em países e regiões (Cancer Research UK).

É notável o encargo da genética individual e familiar na gênese do câncer de laringe, inúmeras pesquisas de casocontrole tem vislumbrado alterações em genes, alelos e proteínas específicas na predição da patologia, principalmente quando aglutinados com outros fatores de risco como idade, sexo, etilismo e tabagismo (Su et al., 2017; Yu et al.,2015; Zhu et al.,2018; Si, Feng \& Han, 2018). Outros estudos também indicam a incumbência de determinados genes e mutações na sobrevivência e prognóstico do câncer e tratamento (Scheel et al.,2016; Coskun et al.,2016).

A exposição a determinados agentes químicos, seja de caráter ocupacional ou não, tem sido aspecto pertinente também no risco de câncer de laringe, tais como exposição a amianto, couro, poeira de madeira, sílica, fuligem de carvão, dentre outros. Em estudo desenvolvido na França, com 244 casos de neoplasia maligna primária de laringe, 187 foram relacionados a exposição ocupacional, na qual a exposição de $80 \%$ dos casos foi o amianto (Grignoux et al.,2019). Outra pesquisa de coorte na Holanda desvendou a associação entre amianto e risco de câncer de laringe, mesotelioma e câncer de pulmão (Offermans et al.,2014).

Não raro, mais da metade dos pacientes adentram ao sistema de saúde nas fases avançadas da doença (Steuer et al.,2017; Markou et al.,2013) em estádio III ou IV, isto deve-se principalmente a demora na procura da instituição de saúde devido a ausência de sintomatologias ou mesmo a inespecificidade dos sinais/sintomas. Dependendo da localização do tumor da laringe há variações na apresentação clínica, progressão e prognóstico da patologia.

Quando presente na região glótica, o tumor apresenta sintomatologias nas fases iniciais, comumente, a rouquidão, aliado a isto, a diminuta drenagem linfática do local retarda a metástase e a destruição das cartilagens e estruturas adjacentes ocorrem tardiamente. O tumor supraglótico, no entanto, adquiri tamanho considerável antes de manifestar sintomatologias, 
além disso, o local é ricamente irrigado pelo sistema linfático o que aumenta as chances de metástases nodais (Markou et al.,2013).

A abordagem terapêutica da neoplasia de laringe está intimamente interligado ao estadiamento, morfologia do tumor e condição clínica do paciente. No Brasil, os estágios iniciais até T2 podem ser tratados com radioterapia e/ou laringectomia parcial, priorizando-se a radioterapia por ser efetiva na maioria dos casos optando a cirurgia em casos de não efetividade do primeiro ou recidiva. A partir do estágio T3 é feito a combinação de cirurgia, radioterapia e/ou quimioterapia, sendo a preservação da laringe dependente de metástase e número de linfonodos acometidos, sendo intensamente discutida a preservação da laringe em determinados casos (American câncer society, 2019; Sociedade Brasileira de oncologia clínica, 2017).

Neste contexto, diante das estatísticas e evidências científicas apresentadas há muitos desafios para enfrentar no que diz respeito ao contexto de neoplasias malignas da laringe, a começar pela necessidade de se difundir informações e ações de combate e prevenção aos fatores de risco para o agravo, anexa aos principais sintomas de alerta. Outro aspecto a ser discutido é a abordagem quanto ao tratamento do paciente e os cuidados as reações adversas e efeitos deste, a qual deve ser feito por uma equipe interdisciplinar, pois sabe-se que o contexto da doença e tratamento pesam na qualidade de vida do paciente (Rinkel et al.,2014; Nocini, Molteni, Mattiuzzi \& Lippi,2020).

Estes fatores quando não bem elucidados ao paciente pode repercutir no abandono do tratamento, uma vez que o paciente pode associa-lo apenas os fatores negativos, por isso, a necessidade de uma equipe atenta e preparada para lidar quanto ao bem estar do paciente e entendimento da terapêutica instituída. Ademais, outras discussões têm sido propostas no contexto desta neoplasia, principalmente no que diz respeito ao envolvimento da genética no desenvolvimento da doença, bem como a necessidade de revisões quanto a preservação da laringe e reabilitação em casos de laringectomia total (Souza et al.,2019; Popescu et al., 2019).

\section{Conclusão}

O perfil de pacientes nesta pesquisa foi sexo masculino, maior de 40 anos, fundamental incompleto, pardo, com histórico atual ou pregresso de etilismo e tabagismo, os quais chegam ao sistema de alta complexidade com câncer localmente avançado na situação com diagnóstico e tratamento encaminhados para radioterapia ou cirurgia, com doença estável após o primeiro tratamento. Parte dos pacientes tinham histórico de exposição ocupacional.

Dessa forma, percebe-se esta neoplasia está fortemente alicerçada na interação dos fatores ambientais (etilismo, tabagismo, exposição ocupacional) e endógenos (genética, histórico familiar). E a sustentação e efetividade do diagnóstico e tratamento está fundamentada na resposta ativa das redes de atenção à saúde.

Por meio destes resultados, é nítido também que o sistema de saúde ainda é tênue para atender aos pacientes em risco ou com a neoplasia de laringe, a começar pelos índices altos de "sem informação" presentes nas bases de dados o que implica na tendência de certas variáveis importantes para a predição do câncer. Ainda observa-se também porcentagem significativa de pacientes que chegam "sem diagnóstico e sem tratamento", evidenciado problemas na rede primária/básica de atendimento.

\section{Referências}

American câncer society. (2019). Treating Laryngeal and Hypopharyngeal Cancers by Stage. https://www.cancer.org.

Brasil. Portaria SAES N. 1399, de 17 de dezembro de 2019 redefine os critérios e parâmetros referenciais para habilitação de estabelecimentos de saúde na alta complexidade em oncologia no âmbito do SUS. http://www.conass.org.br

Ciolofan, M. S., Vlãescu, A. N., Mogoanta, C. A., Ionita, E., Ionitá, C. A. N., et al. (2017). Clinical, Histological and Immunohistochemical Evaluation of Larynx Cancer. Curr Health Sci J. 43(4): 367-375. 10.12865/CHSJ.43.04.14. 
Coskun, C., Verim, A., Farooqi, A. A., Turan, S., Mezani, B., \& Kucukhuseyin, O. (2016). Are There Possible Associations Between MnSOD and GPx1 Gene Variants for Laryngeal Cancer Risk or Disease Progression? Cell Mol Biol (Noisy-le-grand), 30; 62 (5): 25-30. https://pubmed.ncbi.nlm.nih.gov.

Cancer Research UK. Statistics by cancer type: Head and neck cancers statistics. https://www.cancerresearchuk.org.

Deng, Y., Wang, M., Zhou, L., Zheng, Y., Li, N., Tian, T., et al. (2020). Global burden of larynx cancer, 1990-2017: estimates from the global burden of disease 2017 study. Aging (Albany NY). 15; 12(3): 2545-2583. 10.18632/aging.102762.

Grignoux, J., Durand-Moreau, Q., Vongmany, N., Brunel, S., \& Dewitte, J-D. (2019). Work-related Laryngeal Cancer: Trends in France From 2001 to 2016. Eur Ann Otorhinolaryngol Head Neck Dis, 136 (1): 7-12. 10.1016/j.anorl.2018.10.006.

Hashibe, M., Brennan, P., Chuang, S., Boccia, S., Castellsague, X., Chen, C., et al.(2009). Interaction between Tobacco and Alcohol Use and the Risk of Head and Neck Cancer: Pooled Analysis in the International Head and Neck Cancer Epidemiology Consortium. Cancer Epidemiol Biomarkers Prev, 18(2): 541550. 10.1158/1055-9965.EPI-08-0347.

Jayalekshmi, P. A., Nandakumar, A., Akiba, S., Gangadharan, P., \& Koriyama, C. (2013). Associations of Tobacco Use and Alcohol Drinking with Laryngeal and Hypopharyngeal Cancer Risks among Men in Karunagappally, Kerala, India -Karunagappally Cohort Study. PLoS One; 8(8). 10.1371/journal.pone.0073716.

Markou, K., Christoforidou, A., Karasmanis, I., Tsiropoulos, G., Triaridis, S., Constantinidis, I., et al. (2013). Laryngeal cancer: epidemiological data from Northern Greece and review of the literature. Hippokratia. 17 (4): 313-318. https://www.ncbi.nlm.nih.gov/pmc/articles/PMC4097410.

Ministério da Saúde. (2020). Instituto Nacional do câncer José Alencar Gomes de Alencar. Estimativa 2020: incidência de câncer no Brasil. INCA, 2019. https://www.inca.gov.br

Ministério da Saúde. (2020). Departamento de informática do Sistema Único de Saúde. Estatísticas vitais. Mortalidade por câncer de laringe. http://tabnet.datasus.gov.br.

Ministério da Saúde. Instituto Nacional de Câncer (INCA). (2010). Registro hospitalar de câncer: planejamento e gestão. https://www.inca.gov.br

Mulcahy, C. F., Mohamed, A. S. R., Kanwar, A., Hutcheson, K. A., Ghosh, A., \& Vock, D. (2018). Age-adjusted Comorbidity and Survival in Locally Advanced Laryngeal Cancer. Head Neck. 40(9): 2060-2069. 10.1002/hed.25200.

Nocini, R., Molteni, G., Mattiuzzi, C., \& Lippi, G. (2020). Updates on larynx cancer epidemiology. Chin J Cancer Res. 32(1): 18-25. 10.21147/j.issn.10009604.2020.01.03.

Offermans, N. S. M., Vermeulen, R., Burdorf, A., Goldbohm, R. A., Kauppinen, T., Kromhout, H., et al. (2014). Occupational Asbestos Exposure and Risk of Pleural Mesothelioma, Lung Cancer, and Laryngeal Cancer in the Prospective Netherlands Cohort Study. J Occup Environ Med. 56 (1): 6-19. 10.1097/JOM.0000000000000060.

Popescu, B., Ionescu, R., Oasã, I. D., Bertesteanu, S. V. G., Balalau, C., Scaunasu, R. V., et al. (2019). Quality of life challenges for larynx câncer patients. J Clin Invest Surg, 4(1): 27-31. 10.25083/2559.5555/4.1/27.31.

Perdomo, S., Roa, G. M., Brenan, P., Forman, D., Sierra, M. S. (2016). Head and neck cancer burden and preventive measures in Central and South America. Cancer Epidemiology, 44: 43-52. https://doi.org/10.1016/j.canep.2016.03.012.

Rinkel, R. N. P. M., Verdonck-de Leeuw, I. M., van den Brakel, N., Bree, R., Eerenstein, S. E. J., Aaronson, N., \& Leemans, C. R. (2014). Patient-reported symptom questionnaires in laryngeal cancer: Voice, speech and swallowing. Oral onconlogy; 50: 759-64. https://doi.org/10.1016/j.oraloncology.2014.05.009.

Shephard, E. A., Parkinson, M. A., \& Hamilton, W. T. (2019). Recognising laryngeal cancer in primary care: a large case-control study using electronic records. Br J Gen Pract. 69(679):127-133. Recuperado em: Doi: https://doi.org/10.3399/bjgp19X700997.

Su, J., Cui, J., Xue, H-T., Tian, J-H., \& Zhang, J-H. (2017). Study on the Correlation Between CD14 Gene Polymorphism and Susceptibility to Laryngeal Cancer. Eur Rev Med Pharmacol Sci, 21(19):4292-4297. https://pubmed.ncbi.nlm.nih.gov.

Si, F. Z., Feng, Y. K., \& Han, M. (2017). Association between interleukin-17 gene polymorphisms and the risk of laryngeal cancer in a Chinese population. Genet Mol Res., 30; 16 (1). 10.4238/gmr16019076.

Scheel, A., Bellile, M., McHugh, J. B., Walline, H. M., Prince, M. E., Urba, S., et al. (2016). Classification of TP53 Mutations and HPV Predict Survival in Advanced Larynx Cancer. Laryngoscope, Sep; 126(9): E292-E299. 10.1002/lary.25915.

Steuer, C. O., El-Deiry, M., Parks, J. R., Higgins, K. A., \& Saba, N. F. (2017). An update on larynx cancer. Ca cancer J Clin; 67:31-50. https://doi.org/10.3322/caac.21386.

Sociedade Brasileira de oncologia clínica. (2017). Diretrizes para câncer de cabeça e pescoço. https://sboc.org.br.

Souza, F. G. R., Santos, I. C., Freitas, A. S., Thuler, L. C. S., Bergmann, A., Freitas, E. Q., et al. (2019). Comparative analysis of quality of life in advanced laryngeal and oral cancer undergoing extensive surgeries. Head Neck Surg. 47 (3): 1-16. http://dx.doi.org/10.4322/ahns.2018.0029Arch.

Yu, P-G., Chen, W-G., Feng, Q-L., Chen, W., Jiang, M-J., Li, Z-Q. (2015). Association Between CYP1B1 Gene Polymorphisms and Risk Factors and Susceptibility to Laryngeal Cancer. Med Sci Monit., 21: 239-45. 10.12659/MSM.893084.

Zhu, Y., Guo, L., Wang, S., Yu, Q., \& Lu, J. (2018). Association of Smoking and XPG, CYP1A1, OGG1, ERCC5, ERCC1, MMP2, and MMP9 Gene Polymorphisms With the Early Detection and Occurrence of Laryngeal Squamous Carcinoma. J Cancer, 9 (6): 968-977. 10.7150/jca.22841. 